\title{
Desigualdades: subjetividad, otredad y convivencia social en Latinoamérica
}

\author{
MARÍA CRISTINA BAYÓN Y GONZALO A. SARAVÍ
}

Inequalities: Subjectivity, Othering, and Social Interaction

in Latin America

MARIA CRISTINA BAYÓN Universidad Nacional Autónoma de México, Instituto de Investigaciones Sociales,

Ciudad de México, México cristina.bayon@sociales.unam.mx

Gonzalo A. Saraví Centro de Investigaciones y Estudios Superiores en Antropología SocialCiudad de México, México gsaravi@ciesas.edu.mx

Desacatos 59, enero-abril 2019, pp. 8-15 urante las últimas décadas, en las regiones más diversas del planeta, las sociedades contemporáneas han experimentado una ampliación de las brechas que separan a los sectores desfavorecidos de los privilegiados, incluso a las clases medias respecto de unos y otros. Se trata de un fenómeno con implicaciones para el desarrollo, la democracia y el bienestar social que cuestiona los principios de justicia y equidad. Además, y en parte como trasfondo de esas derivaciones, en contextos como Latinoamérica, la persistencia de la desigualdad, sumada a otros procesos seculares, ha implicado una reconfiguración societal en términos de cohesión, integración y convivencia social. La desigualdad social ha pasado a ser un tema de creciente preocupación y relevancia en el campo académico, y en cierta medida, también en el ámbito de la opinión pública y la agenda global.

La segmentación educativa, la segregación residencial, las disparidades en los servicios de salud, la homogeneidad social de los espacios de consumo y entretenimiento, y en general, el distanciamiento y la ausencia de experiencias sociales compartidas son algunas de las expresiones más evidentes de esa fragmentación social. Al mismo tiempo, otras tendencias - como la urbanización, la expansión educativa y del consumo, o las medidas de acción afirmativa - nos empujan hacia una mayor presencia de los "otros" en espacios inesperados. La coexistencia de mundos aislados, vidas paralelas en ámbitos diversos de la vida social, plantea nuevas formas de interacción y sociabilidad, de (no) reconocimiento y (des)encuentro con los otros, de posicionamientos subjetivos y legitimidades sociales, es decir, una reconfiguración de las formas en que nos relacionamos y convivimos en una misma sociedad. Estos aspectos, poco explorados en los estudios sobre desigualdad, en especial en Latinoamérica, pero de creciente interés en las ciencias sociales, constituyen el tema central que se explora en este número de Desacatos. 
Convivencia significa "vivir en compañía de otros", lo que nos invita a preguntarnos cómo interactúa la gente en su vida cotidiana, cómo trata a los otros y se relaciona con ellos, cómo se posiciona de manera subjetiva frente a los demás. Convivencia nos remite al respeto, reconocimiento, solidaridad y empatía; a la capacidad de identificarnos con los otros, comprender sus puntos de vista o simplemente ponernos en sus zapatos (Bayón, 2017). En este sentido, un factor determinante sobre la forma que puede tomar la convivencia social es, precisamente, la existencia o no de experiencias sociales compartidas y su intensidad en espacios como escuelas, hospitales, transporte público, centros de consumo, parques y espacios recreativos, entre muchos ejemplos posibles. La presencia de instituciones comunes y espacios compartidos favorece la interacción con el otro como un semejante con igual valor moral que nosotros y nuestras opciones de vida, promueve los lazos de solidaridad y un sentido de pertenencia común. Sin embargo, en la mayoría de las ciudades latinoamericanas, en las que las brechas sociales históricas se consolidaron o profundizaron en las últimas décadas bajo la influencia del neoliberalismo, estas experiencias compartidas se han debilitado, disminuido o desaparecido, al mismo tiempo que se han constituido o cristalizado ámbitos segregados y homogéneos de sociabilidad. Esto no significa que los encuentros e interacciones cotidianas entre miembros de clases disímiles hayan dejado de existir — en ocasiones, como dijimos, se habrían incrementado-, sino que ocurren desde experiencias socioculturales distantes y con frecuencia recíprocamente invisibilizadas.

En referencia a estos procesos, autores como Dubet (2015: 102) relacionan la intensificación de las desigualdades con una crisis de las solidaridades, es decir, un debilitamiento de los lazos sociales que nos llevan a desear la igualdad de todos, en particular la de aquellos a quienes no conocemos pero reconocemos como semejantes. En tiempos neoliberales, sin embargo, la solidaridad y la responsabilidad compartidas han sido reemplazadas por el individualismo y la responsabilidad personal, sobre todo, por la culpabilización de los más desfavorecidos para explicar su suerte y destino. La responsabilidad personal de los pobres deviene en un discurso moral de la culpa, la falta, que disocia la desigualdad de la estructura social y sus bases materiales. Con este mismo discurso neoliberal hegemónico, los "logros" de los más favorecidos tienden a atribuirse a cualidades o capacidades personales, como el mérito, el esfuerzo o la inteligencia. Así, se despolitiza la condición de clase y la desigualdad se legitima y naturaliza. Esta exaltación del individualismo egoísta sobre la solidaridad conduce a una reconfiguración de fronteras morales que erosiona la confianza y debilita las obligaciones recíprocas (Whitehead, 2018).

Dicho en otros términos, intentamos llamar la atención sobre la confluencia y complementariedad entre un proceso de fragmentación social y un discurso neoliberal que condicionan y reconfiguran los patrones de la convivencia social. En este contexto, los artículos reunidos en este número se proponen explorar, en diversos espacios y situaciones específicas, dos dimensiones esenciales de la convivencia, como la sociabilidad y la subjetivación.

En sus acepciones iniciales y precursoras, el concepto de sociabilidad hacía referencia a un tipo particular de encuentro con el otro, aquel que no tiene otra motivación más que el deseo o disposición de las personas a estar juntas, una acepción muy próxima a la cualidad o acción de "ser sociable" (Simmel, 2002). En este sentido, como esfera de lo social y materia de las ciencias sociales, se asoció a lo lúdico, a las relaciones e interacciones desarrolladas en momentos de recreación, esparcimiento y convivialidad (Di Napoli, 2017). Sin embargo, más allá de estas primeras reflexiones, el análisis de la sociabilidad vuelve a ser relevante hoy en la medida en que nos habilita a indagar en las formas que asume y los patrones que guían el encuentro con otros 
en espacios, relaciones, momentos o situaciones no pautadas de manera estratégica o no institucionalizadas formalmente con anterioridad.

La fragmentación social favorece, y al mismo tiempo es estimulada por, una tendencia creciente a la asociación diferencial, es decir, a asociarnos e interactuar en la vida cotidiana con otros similares a nosotros, algo que Bottero y Prandy (2003) denominan un principio de asociación con los iguales a mí - like me-. El neoliberalismo ha acentuado esta tendencia. La sensación de incomodidad o inseguridad en ciertas interacciones sociales, o la búsqueda de afinidad y compatibilidad en gustos, preferencias o estilos de vida, obstaculizan la construcción de relaciones cercanas entre individuos socialmente distantes. Los contactos entre sectores sociales diferentes, señala Angela Giglia, se evitan "mediante la conformación de ámbitos segregados y la puesta en operación de estrategias sistemáticas de elusión en la vida cotidiana, fundamentadas en el miedo que origina la ciudad: miedo al otro, miedo al caos, miedo a lo imprevisto" (2001: 811). El miedo, la desconfianza y la descalificación moral, incluso estética, del otro se conjugan con la búsqueda de la seguridad — social y subjetiva - provista por una comunidad integrada por gente como uno. La elección de la escuela o universidad, el lugar de residencia, el tipo de transporte, los cines o espacios públicos por los que transitamos suele orientarse por estos principios que reproducen y exacerban las bases materiales de la fragmentación.

La sociabilidad asume así, al menos como expectativa, un carácter con predominio de endogamia o de homogeneidad social: "las personas con las que se es sociable tienden cada vez más a parecerse entre ellas y las situaciones de encuentro se vuelven estereotipadas" (Giglia, 2001: 812). Esto cuestiona de cierta manera el sentido mismo de la sociabilidad, que implica un reconocimiento del otro. A lo largo de los últimos años, múltiples trabajos han analizado los procesos, estrategias y experiencias que conducen al distanciamiento y aislamiento social en varios ámbitos de la vida, como el sistema educativo, la ciudad y el espacio urbano, o el consumo (Carman, Da Cunha y Segura, 2013; Duhau y Giglia, 2008; Caldeira, 2007; Márquez, 2003; Kessler, 2002; Tiramonti y Ziegler, 2008; Alonso, 2004; Bayón y Saraví, 2018). Nosotros también hemos explorado estos temas, ya sea al priorizar las exclusiones recíprocas entre clases antagónicas (Saraví, 2015) o la integración excluyente de los más desfavorecidos (Bayón, 2015). Sin embargo, mucho menos atención ha recibido el análisis de los encuentros con otros distantes a uno mismo, es decir, los encuentros entre desiguales o miembros de clases distintas en el escenario de la fragmentación social. ¿Qué formas adquiere la sociabilidad en estos encuentros, más aún cuando la tendencia es la evitación, el aislamiento y el rechazo del otro? ¿Cuáles son las tensiones, los códigos, las emociones y estigmas puestas en juego en esos momentos de encuentro?

El análisis de la sociabilidad nos permite explorar la calidad de las relaciones sociales (Martuccelli, 2007), pues en ella se disputan las bases del respeto hacia el otro y su reconocimiento como miembro de una misma comunidad. En última instancia, lo que está en juego es hasta qué punto la desigualdad, trasladada a la fragmentación, debilita el tejido social. La calidad de las relaciones sociales se construye sobre cimientos materiales, por lo que la mayor o menor equidad en la distribución de recursos y oportunidades sigue siendo determinante. Sin embargo, junto a las bases materiales relacionadas con la distribución inequitativa de recursos y oportunidades, los modos en que nos relacionamos con los "otros", los tratamos y los representamos tienen un lugar fundamental a la hora de explicar por qué la desigualdad es socialmente aceptada, legitimada y naturalizada.

Las interacciones que nos vinculan a diario están condicionadas por la forma en que definimos y percibimos socialmente a los otros y a nosotros mismos. De manera creciente, en las ciencias sociales se ha reconocido la relevancia de las dimensiones 


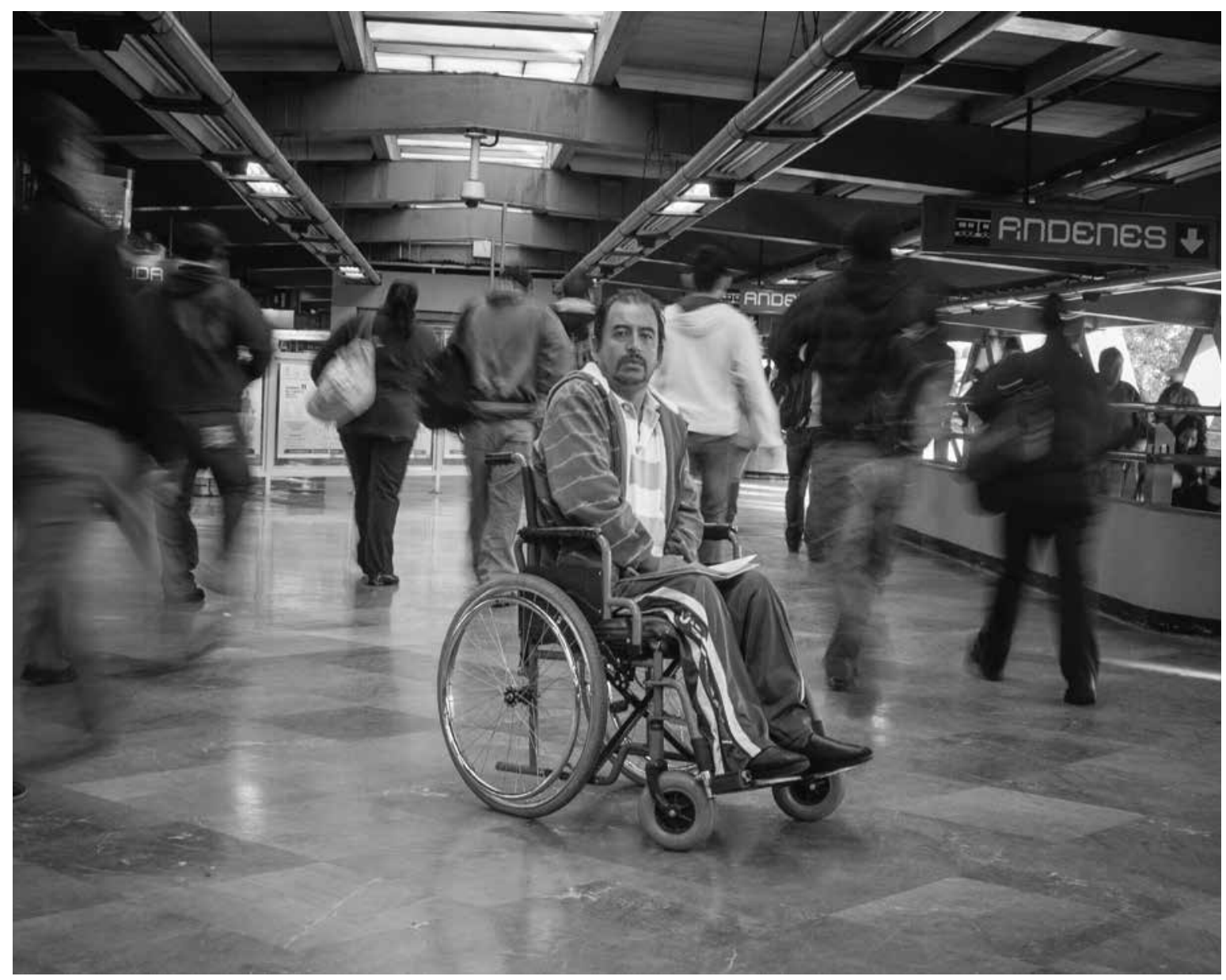

RodRIGo GonZÁlez - Metro de la Ciudad de México: al borde de la crisis, agosto de 2013.

morales y simbólicas en los encuentros e interacciones con los otros, así como en la legitimación y reproducción de la desigualdad y el destino de clase. Es oportuno recordar que la otredad no supone ninguna diferencia innata o esencial, es una construcción social, resultante de sistemas de etiquetamiento y clasificación. Una cuestión central sobre cómo se valoran las distintas clases se refiere, según Bourdieu (1989), a quién tiene acceso al capital simbólico necesario para legitimarse a sí mismo en modos particulares y cuáles son los recursos que se usan para conceptualizar sectores como merecedores o no de reconocimiento y valor social, es decir, el prestigio de unos y la descalificación de otros. Las relaciones de poder y desigualdad entre clases sociales se traducen así en una valoración desigual de sí mismas, de sus gustos y prácticas, de sus barrios y escuelas, de su posición y participación en la sociedad.

El artículo de Kathya Araujo nos invita a reflexionar sobre la centralidad que adquieren las desigualdades en la interacción en la sociedad chilena contemporánea. En "La percepción de las desigualdades: interacciones sociales y procesos sociohistóricos. El caso de Chile”, el foco está puesto en las desigualdades que emergen de las formas como 
somos tratados, ya sea por otros individuos o por las instituciones, en interacciones sociales ordinarias y concretas. A pesar de las profundas desigualdades socioeconómicas que priman en la sociedad chilena, Araujo llama la atención sobre la sensibilidad particular que manifiestan los individuos hacia las desigualdades en el trato, las cuales cuestionarían, precisamente, la valoración y el reconocimiento social de los individuos. A partir de este análisis, es posible pensar en algunas de las contradicciones que tensionan a las sociedades latinoamericanas contemporáneas. Por ejemplo, si bien el principio de igualdad parece ganar peso como pauta que debería regir las interacciones sociales, las tensiones y dilemas que emergen de su transgresión se derivan de la persistencia de estructuras jerárquicas tradicionales e interiorizadas culturalmente que afloran en la sociabilidad cotidiana. Además, este principio o demanda de igualdad en el trato no encuentra, a pesar de su difusión y creciente legitimidad, los canales que lo conduzcan hacia un cuestionamiento sustantivo de las bases materiales de esa desigualdad. Estas últimas, por el contrario, parecen quedar a salvo bajo los principios de un discurso neoliberal, también arraigado, que prioriza la igualdad formal.

Paradojas similares se constatan en la contribución de María José Álvarez Rivadulla, quien encuentra en ellas una expresión más próxima a las tensiones individuales que a las societales. Álvarez Rivadulla aprovecha las oportunidades analíticas que ofrece un programa de acción afirmativa en Colombia, que otorga becas a jóvenes de sectores desfavorecidos, con un desempeño escolar destacado, para que estudien en universidades privadas, por tradición ocupadas por las clases privilegiadas. En “¿Los becados con los becados y los ricos con los ricos? Interacciones entre clases sociales distintas en una universidad de elite", se analiza de manera minuciosa y densa las prácticas, percepciones y emociones de estos jóvenes desfavorecidos, que emergen a partir del encuentro y la convivencia con las elites en un espacio social excepcional. El análisis es muy sugerente, en la medida en que expone las ambivalencias y dilemas de los jóvenes becados en un escenario de igualdad que no termina ni puede disolver las estructuras de desigualdad sobre las que se erige. Allí se ponen en juego las aspiraciones, vergüenzas y tácticas de un grupo particular de jóvenes que se colocan en una situación de bisagra entre sus condiciones precarias de origen y sus expectativas y oportunidades de movilidad individual.

En el artículo de Manuela Camus, "Habitar el privilegio: relaciones sociales en los fraccionamientos cerrados en Guadalajara", las dimensiones morales y simbólicas de la desigualdad afloran en los discursos por los cuales las clases altas mexicanas construyen un nosotros de exclusividad y prestigio que legitima su condición de privilegio. Camus trabaja con un grupo de residentes de fraccionamientos cerrados de clase media-alta y alta en la ciudad de Guadalajara - llamados “cotos” en la localidad-, y analiza a partir de sus relatos las representaciones sobre sí mismos y los otros, en las cuales se ponen en juego asociaciones entre la condición de clase y el valor moral de sus miembros. Uno de los entrevistados dice que vivir en "el fraccionamiento te exige a ser mejor persona” (p. 47). Estas representaciones marcan las relaciones e interacciones con los vecinos — semejantes—, dentro del espacio residencial, y con los "otros" — distintos—, que se ubican más allá de sus muros y barreras. Así como en la sociedad chilena afloran las estructuras jerárquicas tradicionales en los encuentros cotidianos, Camus sugiere para México una sociabilidad en la que se impone un patrón neoestamental que recupera y actualiza estigmatizaciones de clase, raza y género que se arrastran desde el periodo colonial y que naturalizan y dan rigidez a una estructura social profundamente desigual.

La autovaloración de las elites, pero también de amplios sectores de las clases medias, tiene como 
correlato la desvalorización de los "otros", así como los esfuerzos de resistencia y revalorización de lo propio de las clases subalternas (Reygadas, 2015). "La experiencia escolar como experiencia de clase: fronteras morales, estigmas y resistencias" explora estos aspectos. En un ámbito diferente al de los espacios residenciales y desde la mirada de las clases populares, la contribución de Cristina Bayón y Gonzalo A. Saraví se detiene en la estigmatización, la descalificación social y las resistencias. A partir de la experiencia escolar de un grupo de adolescentes de sectores populares de dos escuelas de educación media superior en la Ciudad de México y su área conurbada, analizan las tensiones que emanan de un discurso meritocrático que abre oportunidades y al mismo tiempo legitima jerarquías sociales, la estigmatización y depreciación moral de las escuelas a las que acceden los sectores populares, y las etiquetas y prejuicios que pesan sobre estos alumnos desde la institucionalidad escolar.

A lo largo de su análisis, ponen especial atención en las implicaciones de estas dimensiones simbólicas de la desigualdad sobre la experiencia subjetiva de clase, en cómo estos adolescentes procesan y resisten la descalificación social. Los procesos de clasificación social marcan, y en cierta medida conducen, las prácticas de sociabilidad cotidiana, pero al mismo tiempo impactan en la subjetivación. Los individuos se forman una idea de sí mismos y actúan condicionados por la forma en que son vistos y tratados por otros. El reconocimiento, la valoración y la estima sociales tienen implicaciones sobre múltiples dimensiones de la subjetividad. Por un lado, la clasificación, estigmatización y discriminación dan lugar a un conjunto de emociones y configuraciones subjetivas que tienen un común denominador de clase. La vergüenza, la culpa, la baja autoestima, el conformismo o el resentimiento son algunas de sus expresiones posibles. Por otro lado, no se trata de sujetos pasivos sometidos a etiquetas externas; la necesidad de reconocimiento y autovaloración reclama manifestaciones de resistencia frente al poder simbólico de las clases dominantes y las consecuentes clasificaciones hegemónicas.

El comentario de Gabriel Kessler, con el que cerramos este dosier, reflexiona sobre los desafíos pendientes y una posible agenda de investigación para las ciencias sociales en el campo de los estudios sobre la desigualdad. En sintonía con lo señalado, uno de los desafíos consiste en la necesidad de mantener una perspectiva relacional que nos permita hacer las conexiones entre los hallazgos y contribuciones de los análisis etnográficos micro y meso con sus potenciales implicaciones macro, y viceversa. En los cuatro artículos centrales, el lector encontrará insumos para pensar en algunos de estos vínculos: en una dirección, la legitimidad y la resistencia a la desigualdad tal vez comiencen a gestarse en microescenarios, como los analizados en este número; en otra, las subjetividades — sentimientos y pensamientos - no parecen inmunes a las fuerzas de las configuraciones societales que contienen al sujeto.

En "Algunas reflexiones sobre la agenda de investigación de desigualdades en Latinoamérica", Kessler plantea y propone dos rutas necesarias para nuestras disciplinas: por un lado, revisar, complementar, cuestionar las afirmaciones de una perspectiva económica, aún hoy dominante en la discusión sobre la desigualdad; por el otro, pensar con imaginación nuevas preguntas y enfoques que exploten las potencialidades propias de la antropología y la sociología para contribuir a este debate. Los artículos incluidos en este número de Desacatos representan un esfuerzo por asumir este compromiso. Es claro, y pocos lo cuestionan, que la desigualdad tiene, casi por definición, un impacto directo sobre las condiciones de vida de la población, pero es mucho menos clara, incluso a veces se niega, su 
asociación con el debilitamiento del tejido social y la consecuente emergencia de malestares sociales múltiples. Estos artículos brindan algunas claves para interpretar esa asociación en Latinoamérica con base en estudios empíricos y cualitativos sobre la desigualdad, muy escasos y necesarios para complementar el predominio de los ensayos y las mediciones del problema. La reflexión final de Kessler nos empuja un paso más allá: deja una serie de preguntas nuevas a explorar y paradojas a responder.

Antes de concluir esta presentación, queremos insistir en un aspecto: poner énfasis en la importancia de las dimensiones morales y simbólicas de la desigualdad de clase de ninguna manera supone que ésta se resuelva al reducir el desprecio o el racismo de clase, al favorecer los encuentros con el otro o promover un mayor respeto entre desiguales. Por el contario, las desigualdades de clase persistirán en la medida en que las estructuras que las producen se mantengan intactas. Sin embargo, la falta de reconocimiento agudiza las heridas de clase, legitima las jerarquías sociales y profundiza las brechas de empatía y solidaridad, y se constituye en un nuevo y poderoso obstáculo para alcanzar una sociedad más justa y digna para todos. Tal vez en los microescenarios empiece la transformación de las configuraciones macro. En el camino, además, es válido aspirar a una convivencia más solidaria y con menos humillaciones. D

\section{Bibliografía}

Alonso, Luis Enrique, 2004, "Las políticas del consumo: transformaciones en el proceso de trabajo y fragmentación de los estilos de vida”, en Revista Española de Sociología, vol. 4, pp. 7-50.

Bayón, María Cristina, 2015, La integración excluyente. Experiencias, discursos y representaciones de la pobreza urbana en México, Universidad Nacional Autónoma de México-Instituto de Investigaciones Sociales/Bonilla Artigas Editores, México.

__, 2017, "Vidas paralelas. Desigualdad y convivencia social en sociedades fragmentadas", en Encuentros 2050, núm. 2, pp. 30-33.

Bayón, María Cristina y Gonzalo A. Saraví, 2018, "Place, Class Interaction, and Urban Segregation: Experiencing Inequality in México City”, en Space and Culture, vol. 21, núm. 3, pp. 291-305.

Bottero, Wendy y Kenneth Prandy, 2003, "Social Interaction, Distance and Stratification", en British Journal of Sociology, vol. 54, núm. 2, pp. 177-197.

Bourdieu, Pierre, 1989, "Social Space and Symbolic Power", en Sociological Theory, vol. 7, núm. 1, pp. 14-25.

Caldeira, Teresa, 2007, Ciudad de muros, Gedisa, Barcelona.

Carman, María, Neira Viera da Cunha y Ramiro Segura (coords.), 2013, Segregación y diferencia en la ciudad, Facultad Latinoamericana de Ciencias Sociales-Ecuador/Consejo Latinoamericano de Ciencias Sociales/Ministerio de Desarrollo Urbano y Vivienda, Quito.

Dubet, François, 2015, ¿Por qué preferimos la desigualdad?, Siglo XXI Editores, Buenos Aires.

Duhau, Emilio y Angela Giglia, 2008, Las reglas del desorden. Habitar la metrópoli, Siglo XXI Editores, México.

Giglia, Angela, 2001, "Sociabilidad y megaciudades”, en Estudios Sociológicos, vol. 19, núm. 57, pp. 799-821.

Kessler, Gabriel, 2002, La experiencia escolar fragmentada, Instituto Internacional de Planeamiento de la Educación/Organización de las Naciones Unidas para la Educación, la Ciencia y la Cultura, Buenos Aires.

Márquez, Francisca, 2003, "Identidad y fronteras urbanas en Santiago de Chile”, en Psicologia em Revista, vol. 10, núm. 14, pp. 35-51. Martuccelli, Danilo, 2007, Gramáticas del individuo, Losada, Buenos Aires.

Napoli, Pablo di, 2017, "Sociabilidades juveniles en el ámbito escolar”, en Revista Mexicana de Investigación Educativa, vol. 22, núm. 73, pp. 585-612. 
Reygadas, Luis, 2015, "The Symbolic Dimension of Inequalities", documento de trabajo 78, desiguALdades.net-International Research Network on Interdependent Inequalities in Latin America, Berlín. Disponible en línea: <http://www.desigualdades.net/Working_ Papers/Search-Working-Papers/working-paper-78-_the-symbolic-dimension-of-inequalities_/index.html>.

Saraví, Gonzalo, 2015, Juventudes fragmentadas. Socialización, clase y cultura en la construcción de la desigualdad, Facultad Latinoamericana de Ciencias Sociales-México/Centro de Investigaciones y Estudios Superiores en Antropología Social, México.

Simmel, George, 2002, Cuestiones fundamentales de sociología, Gedisa, Barcelona.

Tiramonti, Guillermina y Sandra Ziegler, 2008, La educación de las elites. Aspiraciones, estrategias y oportunidades, Paidós, Buenos Aires. Whitehead, Philip, 2018, Demonising the Other. The Criminalisation of Morality, Policy Press, Bristol. 